\title{
Planejamento estratégico situacional elaborado em uma unidade de saúde da família: um relato de experiência
}

RESUMO | Este artigo tem como objetivo relatar a elaboração de um Planejamento Estratégico Situacional (PES) realizado por profissionais residentes. Trata-se de um relato de experiência onde os encontros realizados para a elaboração do PES iniciou no dia 1 de outubro de 2016 e se deu até 31 de janeiro de 2017. Os dados foram coletados nos meses de outubro a novembro de 2016 em seis momentos, utilizou como metodologias as estratégias de rodas de conversa e exposição dialogada. O alto índice de pacientes com hipertensão arterial e diabetes mellitus foi o resulto encontrado, tendo como discursão a garantia do acompanhamento integral deste público supracitado e a baixa adesão as consultas de hipertensão arterial e diabetes mellitus. Para os profissionais residentes a realização do PES foi essencial para formação profissional, visto que essa ferramenta de trabalho despertou uma reflexão sobre as formas de assistência prestada pelos profissionais da Atenção Básica.

Palavras-chaves: Planejamento em saúde; Atenção Básica; Doenças Crônicas.

\begin{abstract}
This article aims to report the development of a Situational Strategic Planning (PES) carried out by resident professionals. This is an experience report where the meetings held for the preparation of the PES started on October 1,2016 and took place until January 31, 2017. Data were collected from October to November 2016 in six moments, used as methodologies the strategies of conversation circles and dialogued exposition. The high rate of patients with arterial hypertension and diabetes mellitus was the result found, having as a discourse the guarantee of full monitoring of this aforementioned public and the low adherence to hypertension and diabetes mellitus consultations. For resident professionals, the performance of the PES was essential for professional training, since this work tool aroused a reflection on the forms of assistance provided by Primary Care professionals.
\end{abstract}

Keywords: Health planning; Basic Attention; Chronic diseases.

RESUMEN | Este artículo tiene como objetivo informar del desarrollo de una Planificación Estratégica Situacional (PES) realizada por profesionales residentes. Se trata de un relato de experiencia donde las reuniones realizadas para la elaboración del PES comenzaron el 1 de octubre de 2016 y se llevaron a cabo hasta el 31 de enero de 2017. Se recolectaron datos de octubre a noviembre de 2016 en seis momentos, se utilizaron como metodologías las estrategias de los círculos de conversación y exposición dialogada. La alta tasa de pacientes con hipertensión arterial y diabetes mellitus fue el resultado encontrado, teniendo como discurso la garantía de un seguimiento completo de este público mencionado y la baja adherencia a las consultas de hipertensión arterial y diabetes mellitus. Para los profesionales residentes, la actuación de los SPE fue fundamental para la formación profesional, ya que esta herramienta de trabajo suscitó una reflexión sobre las formas de atención que prestan los profesionales de Atención Primaria.

Palabras claves: Planificación de la salud; Atención básica; Enfermedades crónicas.

\section{Jaiane Façanha Lessa}

Bacharel em Enfermagem - Universidade

Regional do Cariri - URCA.

ORCID: 0000-0003-2496-7649

Recebido em: 23/01/2021

Aprovado em: 02/02/2021

INTRODUÇÃO

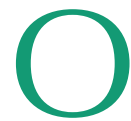

planejamento é definido como uma estratégia situacional caracterizado como um método flexível, capaz de se adequar rapidamente as necessidades locais por meio de definição de problemas e determinações da causalidade e da consequência dos mesmos. ${ }^{1}$
O modelo de Planejamento Estratégico Situacional (PES) surge no âmbito mais geral do planejamento econômico-social e vem sendo crescentemente adaptado e utilizado nas áreas como saúde e educação. O PES é um método de planejamento por problemas e trata, principalmente, dos problemas mal estruturados e complexos, para os quais existe solução normativa ou previamente conhecida como no caso daqueles bem estruturados. ${ }^{2}$

O Indicador de saúde é uma unidade de medida caracterizada como instrumento de mensuração para gerenciar, avaliar e planejar as ações, possibilitando então mudanças destas ações e, garantindo a melhora de determinada variante. ${ }^{3}$
Os indicadores utilizados nas Unidades de Saúde da Família traduzem quantitativamente os problemas encontrados e alertam o profissional da necessidade de se realizar um planejamento específico para a mudança de tal indicador. ${ }^{4}$

A hipertensão arterial sistêmica (HAS) é uma condição clínica multifatorial caracterizada por níveis elevados e sustentados de pressão arterial - PA (PA $\geq 140$ $\times 90 \mathrm{mmHg}$ ). Associa-se, frequentemente, às alterações funcionais e/ou estruturais dos órgãos-alvo (coração, encéfalo, rins e vasos sanguíneos) e às alterações metabólicas, com aumento do risco de eventos cardiovasculares fatais e não fatais. ${ }^{5}$

A Hipertensão Arterial além de ser 
causa direta de cardiopatia hipertensiva, é fator de risco para doenças decorrentes de aterosclerose e trombose, que se manifestam, predominantemente, por doença isquêmica cardíaca, cerebrovascular, vascular periférica e renal. ${ }^{5}$

O termo "Diabetes Mellitus" (DM) refere-se a um transtorno metabólico de etiologias heterogêneas, caracterizado por hiperglicemia resultantes de defeitos da secreção e/ou da ação da insulina. É um problema de saúde pública caracterizado por ser uma condição sensível à Atenção Primária, neste sentido, estudos demonstram que um acompanhamento adequado neste nível de saúde evita-se hospitalizações e mortes por complicações cardiovasculares e cerebrovasculares. ${ }^{6}$

$\mathrm{O}$ interesse pela temática surgiu a partir do momento em que foi disparado aos profissionais residentes a realização da construção de um PES, visto que nos momentos da elaboração e execução deste instrumento, percebeu-se a importância desta ferramenta a ser utilizada no território como um serviço potencial para detecção e avaliação dos principais fatores que afetam o proceso de saúde-doença de uma comunidade.

Este artigo é de grande relevância, pois com o resultado dele, pretende-se destacar a importância da elaboração de um PES na Atenção Básica com a perspectiva de despertar em todos os atores que compõem a equipe de saúde da família em realizarem uma análise crítica dos problemas existentes no território para que posteriormente possam traçar um plano de ação que tenha como objetivo solucionar ou minimizar esses entraves de saúde existentes dentro da comunidade.

Neste sentido, o presente artigo tem como objetivo relatar a elaboração de um Planejamento Estratégico Situacional realizado por profissionais residentes em um município no interior do estado do Ceará.

\section{MÉTODO}

Trata-se de um relato de experiência que buscou descrever encontros de ela- boração e execução de um Planejamento Estratégico Situacional. O relato de experiência é considerado um instrumento de pesquisa que possibilita ao pesquisador analisar determinadas ações que foram vivenciadas trazendo consigo as descrições dos momentos dando ênfase nas singularidades existentes do momento, sendo assim, descrevendo as principais características que se quer abordar durante o desenvolvimento do estudo. ${ }^{7}$

O município de Quixeramobim está localizado na Mesorregião dos Sertões Cearenses. É a segunda maior cidade do sertão central, com uma população residente estimada em 75565 habitantes. ${ }^{8}$

O cenário da experiência foi em uma unidade Básica de Saúde na zona urbana do município de Quixeramobim composta por uma equipe multidisciplinar com um médico, duas técnicas de enfermagem, uma enfermeira, odontólogo e uma técnica em saúde bucal, atendente, agentes comunitários de saúde e uma equipe NASF residente da Escola de Saúde Pública do Ceará (um Fisioterapeuta, Psicóloga, Nutricionista e Assistente Social).

O período de elaboração e execução do PES iniciou-se no dia 1 de outubro de 2016 e se deu até 31 de janeiro de 2017. Os encontros forma realizados de acordo com as demandas existentes durante a execução e análise da proposta do PES, se utilizou metodologias ativas dentro da proposta das rodas de conversa.

Inicialmente foi explicado para equipe da unidade básica de saúde a proposta principal da realização dos encontros e que a participação de todos os profissionais seria fundamental neste processo, além disso ficou acordado entre os mesmos que o local seria na própria unidade de saúde já que todos os profissionais tem o acesso e o dia e horário fechados foram ás 09:00 da manhã ás sextas-feiras, ficou estabelecido estes critérios tendo em vista que no final da semana para a equipe a demanda diminui um pouco e assim tendo uma disponibilidade de tempo maior para a realização dos encontros.

Os dados foram coletados nos me- ses que compreenderam de outubro a novembro de 2016, os profissionais que participaram do estudo no decorrer dos seis encontros: foram compostas pelas enfermeiras ( 2 do município e 2 residentes), dois técnicos de enfermagem, uma médica, NASF Residência (Fisioterapeuta e Psicóloga), agentes comunitários de saúde, preceptoria de campo e núcleo e usuários e alguns setores que compõem as Redes de atenção à Saúde.

Durante a execução dos seis encontros para que os dados fossem coletados, se utilizou como metodologias as estratégias de rodas de conversa e exposição dialogada, onde através da avaliação conseguiu definir-se o público alvo que foi compreendida pelos indivíduos portadores de hipertensão arterial e diabetes mellitus estando na faixa etária de 25 a 90 anos.

\section{RESULTADOS E DISCUSSÃO}

Para que fosse colocado em prática o Planejamento Estratégico Situacional (PES) se fez necessário conhecer as Redes de Atenção à Saúde do município de Quixeramobim, bem como os dispositivos sociais atuantes dentro da comunidade.

Neste sentido, a rede assistencial do município é organizada através dos serviços que a compõem como o Núcleo de Apoio à Saúde da Família (NASF), Policlínica de Quixeramobim, Laboratório de Análises Clínicas, Centro de Atenção Psicossocial de Quixeramobim (CAPS Geral II), Centro de Atenção Psicossocial Álcool e outras Drogas - Alpendre, Hospital Regional Dr. Pontes Neto (HRDPN), Hospital Infantil Nossa Senhora do Perpétuo Socorro, Centro de Referência de Saúde do Trabalhador (CEREST), Centro de Atenção Integrada de Quixeramobim (CAIQ), Coordenação de Assistência Farmacêutica (CAF), Serviço de Atenção Domiciliar (SAD), Núcleo de Controle, Avaliação, Regulação e Auditoria (NUCARA), Centro de Especialidades Odontológicas (CEO) Regional, Secretaria Municipal de Saúde (SMS), Vigilância Epidemiológica, SAMU. 
A partir deste ponto da análise dos serviços de saúde ofertados pelo município e bem como os serviços de apoio dentro da comunidade que poderiam fazer parte da elaboração e execução do Planejamento Estratégico Situacional (PES) ocorreu à definição dos atores que participaram do planejamento, onde foi composta pelas enfermeiras (2 do município e 2 residentes), dois técnicos de enfermagem, uma médica, NASF Residência (Fisioterapeuta e Psicóloga), agentes comunitários de saúde, preceptoria de campo e núcleo e usuários e alguns setores que compõem as Redes de atenção à Saúde.

No segundo passo foi realizado uma reunião com todos os atores envolvidos e listados os problemas da unidade e os determinantes de cada um, ficando definido realizar um planejamento estratégico situacional em saúde aos pacientes com hipertensão e diabetes. A equipe percebeu que muitos pacientes hipertensos e diabéticos não estavam sendo acompanhados, procuravam a unidade apenas com o intuito de renovar a receita ou quando já apresentavam alguma complicação.

O terceiro passo foi uma roda de conversa com alguns usuários, no qual foi percebido o déficit de conhecimento com o manejo clínico das citadas condições crônicas, bem como as formas de prevenção de suas complicações. A seguir foi realizado a identificação do problema de situação de saúde, "alto índice de pacientes com hipertensão arterial e Diabetes mellitus" e o problema de produção em saúde "baixa adesão as con- sultas de hipertensão arterial e Diabetes mellitus". Essa etapa foi mais crítica para ser executada, pois os membros tiveram um olhar diferenciado, sendo analisado os gráficos da sala de situação em saúde realizado no território, e assim foi discutido o problema, e construído um consolidado em forma de check-list, contendo a relação de pacientes hipertensos e diabéticos com suas co-morbidades e que foram entregues a cada Agentes Comunitários de Saúde (ACS), para realizar a identificação dos pacientes com esse perfil no território.

Neste sentido, o consolidado foi composto da seguinte forma: identificação do usuário, data nascimento, idade, e se apresentava algum dos seguintes problemas de saúde hipertensão, diabetes, obesidade, tabagista, sedentário, co-morbidades (Acidente Vascular Cerebral, nefropatia e retinopatia) e acompanhamento em unidade especializada.

No quarto passo os Agentes Comunitários de Saúde (ACS) a partir das visitas domiciliares realizaram a busca ativa dos pacientes com base no preenchimento da planilha. Concomitantemente, foi realizado o quinto passo, onde realizamos um encontro através de uma roda de conversa com a gestão municipal de saúde, com o intuito de socializarmos os achados até então encontrados durante a construção do Planejamento Estratégico Situacional (PES), bem como a Coordenadora de Educação Permanente teve ampla participação neste processo.

No sexto passo ocorreu uma reunião entre residentes e a equipe de saúde da família para construção do plano de ação de enfrentamento da problemática identificada. Para a construção foi utilizado como orientação os cadernos do Ministério da Saúde de Hipertensão e Diabetes e os textos disponibilizados para o módulo de Planejamento. No território da unidade encontra-se um centro de convivência do idoso - $\mathrm{CCl}$ que funciona as terças e quintas no período da manhã, com objetivo de entretenimento e socialização aos idosos e desde o início da Residência tem-se mostrado um grande parceiro para o desenvolvimento das ações.

Desta forma, foi incluído o Centro de Convivência do Idoso $(\mathrm{CCl})$ nas atividades de educação em saúde para abordagem da população idosa como uma das intervenções, visto que é a faixa etária mais acometida pelas doenças crônicas no território. Com o plano a equipe terá subsídios que irão orientar as próximas ações para realizar o cuidado aos pacientes, a fim de manter o controle efetivo das citadas condições crônicas e evitar previamente complicações.

No plano de ação os problemas identificados foram o alto índice de pacientes com hipertensão arterial e diabetes mellitus tendo como resultado esperado garantir acompanhamento integral deste público alvo supracitado e a baixa adesão as consultas de hipertensão arterial e diabetes mellitus com resultado esperado que os pacientes desenvolvam autonomia para o autocuidado, sendo destacado nos quadros 1 e 2 as etapas do processo de operacionalização de cada problema citado.

Contudo, no decorrer da implementação do PES pode-se perceber que é um instrumento de trabalho que proporciona

Quadro 1: Operacionalização do planejamento ao alto índice de pacientes com hipertensão arterial e diabetes mellitus. Quixeramobim, Ceará, Brasil, 2016.

\begin{tabular}{|l|c|c|c|}
\hline AÇÃO & RECURSOS & RESPONSÁVEL & PERÍODO \\
\hline $\begin{array}{l}\text { Realizar o levantamento dos pacientes } \\
\text { com HAS e DM e co-morbidades }\end{array}$ & $\begin{array}{c}\text { Visitas ACS Check-list do Processo } \\
\text { saúde-doença }\end{array}$ & $\begin{array}{c}\text { Agentes comunitários de } \\
\text { saúde }\end{array}$ & Outubro de 2016 \\
\hline $\begin{array}{l}\text { Realizar estratificação de risco dos } \\
\text { pacientes }\end{array}$ & $\begin{array}{c}\text { Protocolo de estratificação de risco } \\
\text { (nome do protocolo) }\end{array}$ & $\begin{array}{c}\text { Médica, enfermeiras, cirurgião } \\
\text { dentista e residentes }\end{array}$ & 14 de novembro de 2016 \\
\hline $\begin{array}{l}\text { Realizar busca ativa dos pacientes com } \\
\text { HAS e DM associadas + co-morbidades }\end{array}$ & Visitas ACS Marcação de consultas & ACS Recepção & Cuidado continuado \\
\hline
\end{tabular}




\begin{tabular}{|l|c|c|c|}
\hline Realizar acompanhamento dos pacientes & Visitas ACS Atendimento & $\begin{array}{c}\text { Médica, enfermeiras, NASF e } \\
\text { residência, ACS }\end{array}$ & Cuidado continuado \\
\hline $\begin{array}{l}\text { Oferecer exames e consultas especializa- } \\
\text { das, se necessário }\end{array}$ & $\begin{array}{c}\text { Rede de referência e apoio diag- } \\
\text { nóstico }\end{array}$ & SMS & Cuidado continuado \\
\hline
\end{tabular}

Quadro 2- Operacionalização do planejamento a baixa adesão as consultas de hipertensão arterial e diabetes mellitus. Quixeramobim, Ceará, Brasil, 2016.

\begin{tabular}{|l|c|c|c|}
\hline AÇÃO & RECURSOS & RESPONSÁVEL & PERÍODO \\
\hline Realizar educação em saúde no CCI & $\begin{array}{c}\text { Definir de acordo com atividade a ser } \\
\text { realizada }\end{array}$ & $\begin{array}{c}\text { Equipe saúde da família e } \\
\text { residência }\end{array}$ & Mensal \\
\hline Criar grupo de doenças crônicas na ADS & $\begin{array}{c}\text { Definir de acordo com atividade a ser } \\
\text { realizada }\end{array}$ & $\begin{array}{c}\text { Equipe saúde da família, NASF } \\
\text { e residência }\end{array}$ & Mensal \\
\hline Realizar sala de espera & $\begin{array}{c}\text { Definir de acordo com atividade a ser } \\
\text { realizada }\end{array}$ & $\begin{array}{c}\text { Equipe saúde da família, NASF } \\
\text { e residência }\end{array}$ & De acordo com a demanda \\
\hline
\end{tabular}

aos atores envolvidos avaliar e reorganizar as metas e os resultados que não puderam ser alcançados, pois este fato ocorre devido que o território é um espaço vivo e dinâmico sujeito as mudanças e transformações no que diz respeito ao processo de saúde doença da população adstrita.

Portanto, o PES é uma ferramenta que viabiliza a identificação das potencialidades e dificuldades locais do espaço de atuação da equipe de saúde, onde o enfoque situacional garante a participação efetiva de todos os atores envolvidos nesse processo. ${ }^{9}$

Se fez necessário pontuar que o acompanhamento da implementação das ações que foram realizadas serão constantes, visto que foi pactuado com a equipe da unidade de saúde a continuação da execução do Planejamento Estratégico Situacional, pois é de suma importância que os atores envolvidos possam avaliar e analisar se as atividades até então realizadas alcançaram ou não os objetivos traçados para que a partir deste pressuposto consigam identificar as mudanças essenciais para melhorar a qualidade de vida da população que compõem o território.

\section{CONCLUSÃO}

Após elencarmos os indicadores que seriam trabalhados nesse Planejamento foram vários passos até chegarmos a elaborar um plano de intervenção. Foram vários atores envolvidos porque quando se trata de saúde, na maioria das vezes precisamos acionar a rede visto que são vários os determinantes de saúde.

Essa atividade foi extremamente importante, pois disparou em todos os atores envolvidos a importância de se ter o olhar mais aguçado para as necessidades da comunidade na qual estamos inseridos e que é fundamental planejar ações voltadas para as problemáticas reais do território.

Neste sentido, aos profissionais residentes as etapas vividas na construção e desdobramento na realização do PES foi essencial para formação profissional, visto que essa ferramenta de trabalho despertou nos mesmos uma reflexão sobre as formas de assistência prestada pelos profissionais na Unidade Básica de Saúde, pois os cuidados e as ações realizadas não tinham uma assistência continuada.

\section{Referências}

1.Sampaio RF, Mancini MC, Fonseca ST. Produção científica e atuação profissional: aspectos que limitam essa integração na Fisioterapia e na terapia ocupacional. Revista Brasileira, Fisioter,2002;6(3): 113-118.

2.Artmann E. 0 planejamento estratégico situacional no nível local: um instrumento a favor da visão multissetorial. Cadernos da Oficina Social 3: Série Desenvolvimento Local. Rio de Janeiro: Coppe/UFRJ; 2000.

3.Reis LG. Produção de monografia as teorias a prática o método educar pela pesquisa. Brasília - DF. SENAC. 2008.

4.Santos RGS, Silva JN, Cavalcante VS, Santos CQ, Ferreira T F. Planejamento estratégico elaborado em uma clínica da família para a população idosa? Um relato de experiência. Revista. Nursing. Säo Paulo, 17(223), 1311-1315, jun.2016.

5.Brasil. Ministério da Saúde. Secretaria de Atenção à Saúde. Departamento de Atenção Básica. Estratégias para o cuidado da pessoa com doença crônica:
Hipertensão Arterial Sistêmica. Brasília: Ministério da Saúde, 2013. 6.Brasil. Ministério da Saúde. Secretaria de Atenção à Saúde. Departamento de Atenção Básica. Estratégias para o cuidado da pessoa com doença crônica: Diabetes mellitus. Brasília: Ministério da Saúde, 2013.

7. Cavalcante BLL, Lima UTS. Relato de experiência de uma estudante de Enfermagem em um consultório especializado em tratamento de feridas. J Nurs Health, Pelotas (RS) 2012 jan/jun;1(2):94-103.

8.lbge - INSTITUTO BRASILEIRO DE GEOGRAFIA E ESTATÍ́STICA. Senso Demográfico 2010 (Resultados iniciais). Available from: http://www.ibge.gov.br/ home/.

9. De Menezes, Anderson Rio Branco et al. Planejamento estratégico situacional: relato de experiência vivida em um hospital de ensino. In: $11^{\circ}$ Congresso Internacional da Rede Unida. 2014. 